PM 95-36

\title{
Variational solution of the Gross-Neveu model at finite temperature in the large $N$ limit.
}

\author{
B. Bellet, P. Garcia, F. Geniet and M.B. Pinto \\ Laboratoire de Physique Mathématique, Université de Montpellier II \\ CNRS-URA 768, 34095 Montpellier Cedex 05, France
}

\begin{abstract}
We use an improved nonperturbative variational method to investigate the phase transition of the Gross-Neveu model. It is shown that the variational procedure can be generalized to the finite temperature case. The large $N$ result for the phase transition is correctly reproduced.
\end{abstract}




\section{INTRODUCTION}

A survey of the literature concerning analytical nonperturbative methods shows a growing interest in variational methods such as the gaussian approximation [1] and the optimized linear $\delta$ expansion [2]. Recently, an alternative variational method [3] has been improved and successfully tested in the Gross-Neveu (GN) model [4] both in the large [5] and finite [6] $N$ limits. Basically this new approximation differs from the existing optimized linear $\delta$ expansion in two aspects: it provides a clear way of carrying on the renormalization program of the theory and improves the calculation with a simple but powerful analytical method which allows to attain arbitrarily large and even infinite order of perturbation in the parameter $\delta$. Our goal is to show that this calculational scheme, especially the variational procedure, still works when external parameters such as the temperature are considered. From the renormalization point of view the temperature does not create any new difficulties and the elimination of divergencies can be carried out exactly as at zero temperature [5]. However, previous experience with the related $\delta$ expansion shows that the application of variational methods to the study of phase transitions is not necessarily straightforward [7].

Here, our aim is to check the ability of the variational procedure used in Ref. [5] in generating accurate results for the phase transition, especially near the critical temperature. In this first finite temperature application we restrict ourselves to the large $N$ limit of the GN model where the calculational scheme can be set up more clearly. For comparison we consider the results of Refs. [8] and [9] as being "exact" in the large $N$ limit, despite their disagreement with existing theorems [10] for symmetry breaking/restoration in $1+1$ dimensions. This interesting problem, which has been studied by several authors using different models [11-15], will be explicitly treated in an extension of the present work [16].

In the next section we review the usual large $N$ result for the temperature dependent fermionic mass and perform the variational calculation to lowest orders. We shall see that, as in the zero temperature case, the variational procedure fails. In Section III we follow Ref. [5] to rectify the situation by performing the variational calculation to all orders. When this 
is done the large $N$ result is completely reproduced. The conclusions and future perspectives are presented in section IV.

\section{THE VARIATIONAL CALCULATION TO LOWEST ORDERS}

The variational calculation starts with the addition of an arbitrary bare mass $\left(m_{0}\right)$ to the original massless Gross-Neveu [4]

$$
\mathcal{L}=i \sum_{i=1}^{N} \bar{\psi}_{i} \not \partial \psi_{i}+m_{0} \sum_{i=1}^{N} \bar{\psi}_{i} \psi_{i}+\frac{g_{0}^{2}}{2}\left(\sum_{i=1}^{N} \bar{\psi}_{i} \psi_{i}\right)^{2}
$$

where $g_{0}$ is the bare coupling constant (in the following we shall suppress the summation over the index $i$ ). The relation to the linear $\delta$ expansion and other variational methods becomes clear by performing the substitutions

$$
\begin{gathered}
m_{0} \rightarrow m_{0}(1-\delta), \\
g_{0}^{2} \rightarrow \delta g_{0}^{2} .
\end{gathered}
$$

These will be done at a later stage in order to avoid the explicit evaluation of Feynman graphs which differ only by $\delta m_{0}$ insertions. To perform finite temperature calculations in the imaginary time formalism one does the following substitutions [17]

$$
\begin{gathered}
\int \frac{d p_{0}}{2 \pi} \rightarrow i T \sum_{n}, \\
p_{0} \rightarrow i \omega_{n},
\end{gathered}
$$

where for fermions

$$
\omega_{n}=T(2 n+1) \pi
$$

The sum over Matsubara's frequencies can be performed with

$$
T \sum_{n} \ln \left(\omega_{n}^{2}+m_{0}^{2}\right)=E+2 T \ln [1+\exp (-E / T)]+c
$$


where $\mathrm{c}$ is a $E$-independent constant. In $1+1$ dimensions

$$
E=\left(p_{1}^{2}+m_{0}^{2}\right)^{\frac{1}{2}}
$$

The remaining space integral is evaluated in $1-\epsilon$ dimensions using conventional dimensional regularization techniques.

In the large $N$ limit a perturbative calculation of the fermionic mass $\left(M_{F}\right)$ to $\mathrm{O}\left(g_{0}^{4}\right)$ yields

$$
M_{F}^{(2)}=m_{F}^{(0)}+g_{0}^{2} m_{F}^{(1)}+g_{0}^{4} m_{F}^{(2)}+\mathrm{O}\left(g_{0}^{6}\right)
$$

where $m_{F}^{(0)}=m_{0}$,

$$
m_{F}^{(1)}=\frac{N}{2 \pi} m_{0}^{1-\epsilon}\left[(4 \pi)^{\frac{\epsilon}{2}} \Gamma(\epsilon / 2)-4 I_{1}^{T}\left(y_{0}\right)\right]
$$

and

$$
\begin{aligned}
m_{F}^{(2)}= & \frac{N^{2}}{4 \pi^{2}} m_{0}^{1-2 \epsilon}\left\{(4 \pi)^{\epsilon} \Gamma^{2}(\epsilon / 2)(1-\epsilon)\right. \\
& +4(4 \pi)^{\frac{\epsilon}{2}} \Gamma(\epsilon / 2)\left[(3 \epsilon-2) I_{1}^{T}\left(y_{0}\right)+\left(m_{0} / T\right)^{2}(1-\epsilon)\left(I_{2}^{T}\left(y_{0}\right)+I_{3}^{T}\left(y_{0}\right)\right)\right] \\
& \left.+16 I_{1}^{T}\left(y_{0}\right)\left[I_{1}^{T}\left(y_{0}\right)-\left(m_{0} / T\right)^{2}\left(I_{2}^{T}\left(y_{0}\right)+I_{3}^{T}\left(y_{0}\right)\right)\right]\right\} .
\end{aligned}
$$

The temperature dependent integrals are

$$
\begin{aligned}
& I_{1}^{T}\left(y_{0}\right)=\frac{\pi^{\frac{1-\epsilon}{2}}}{\Gamma(1 / 2-\epsilon / 2)}\left(2 \pi y_{0}\right)^{\epsilon} \int_{0}^{\infty} d x \frac{x^{-\epsilon}}{\left(x^{2}+y_{0}^{2}\right)^{\frac{1}{2}}\left[1+\exp \left(x^{2}+y_{0}^{2}\right)^{\frac{1}{2}}\right]} \\
& I_{2}^{T}\left(y_{0}\right)=\frac{\pi^{\frac{1-\epsilon}{2}}}{\Gamma(1 / 2-\epsilon / 2)}\left(2 \pi y_{0}\right)^{\epsilon} \int_{0}^{\infty} d x \frac{x^{-\epsilon}}{\left(x^{2}+y_{0}^{2}\right)^{\frac{3}{2}}\left[1+\exp \left(x^{2}+y_{0}^{2}\right)^{\frac{1}{2}}\right]}
\end{aligned}
$$

and

$$
I_{3}^{T}\left(y_{0}\right)=\frac{\pi^{\frac{1-\epsilon}{2}}}{\Gamma(1 / 2-\epsilon / 2)}\left(2 \pi y_{0}\right)^{\epsilon} \int_{0}^{\infty} d x \frac{x^{-\epsilon} \exp \left(x^{2}+y_{0}^{2}\right)^{\frac{1}{2}}}{\left(x^{2}+y_{0}^{2}\right)\left[1+\exp \left(x^{2}+y_{0}^{2}\right)^{\frac{1}{2}}\right]^{2}}
$$

with $y_{0}=m_{0} / T$ and $x=p_{1} / T$. These integrals are related to each other via

$$
--2 y_{0}^{\epsilon} \frac{\partial\left(y_{0}^{-\epsilon} I_{1}^{T}\right)}{\partial y_{0}^{2}}=I_{2}^{T}+I_{3}^{T}
$$


The nonperturbative large $N$ calculation consists in the evaluation of all cactus diagrams which can be summed up as

$$
M_{F}=m_{0}\left\{1-\frac{N}{2 \pi} g_{0}^{2} M_{F}^{-\epsilon}\left[(4 \pi)^{\frac{\epsilon}{2}} \Gamma(\epsilon / 2)-4 I_{1}^{T}\left(y_{F}\right)\right]\right\}^{-1}
$$

where $y_{F}=M_{F} / T$. The bare and renormalized parameters are related via

$$
m_{0}=Z_{m} m
$$

and

$$
g_{0}^{2}=Z_{g} g^{2} \mu^{\epsilon}
$$

where $\mu$ is the arbitrary scale introduced by dimensional regularization. The renormalization constants are

$$
Z_{m}=Z_{g}=\left[1+\frac{g^{2} N}{\pi \epsilon}\right]^{-1}
$$

Substituting Eqs. (17) and (18) into Eq. (16) yields the finite expression for the dimensionless quantity $M_{F} / \Lambda \overline{M S}$

$$
\frac{M_{F}}{\Lambda_{\overline{M S}}}=\frac{m}{\Lambda_{\overline{M S}}}\left\{1+\frac{N}{\pi} g^{2}\left[\ln \left(\frac{M_{F}}{\bar{\mu}}\right)+2 I_{1}^{T}\left(y_{F}\right)\right]\right\}^{-1},
$$

where $\Lambda_{\overline{M S}}=\bar{\mu} \exp \left[-\pi /\left(N g^{2}\right)\right]$ and $\bar{\mu}=\mu(4 \pi)^{\frac{1}{2}} \exp \left(-\gamma_{E} / 2\right)$ with $\gamma_{E}=0.577215$. Equation (20) satisfies the $\mathrm{RG}$ equation

$$
\mu \frac{d}{d \mu} M_{F}=\left(\mu \frac{\partial}{\partial \mu}+g \beta(g) \frac{\partial}{\partial g}-m \gamma_{m}(g) \frac{\partial}{\partial m}\right) M_{F}=0
$$

where

$$
\beta(g)=-\frac{N}{2 \pi} g^{2} \text { and } \gamma_{m}(g)=\frac{N}{\pi} g^{2}
$$

Then, in the chiral limit $(m=0)$, the finite temperature mass gap equation is given by

$$
M_{F}(T)=M_{F}(0) \exp \left[-2 I_{1}^{T}\left(y_{F}\right)\right]
$$


where $M_{F}(0)=\Lambda_{\overline{M S}}$ is the renormalized fermion mass, generated through dynamical symmetry breaking at $T=0$. This is just the large $N$ result which reproduces the phase diagram for the fermion mass [8].

The variational calculation starts with the substitutions Eqs. (22) and (3). With this procedure Eq. (1) interpolates between the original massless GN model (at $\delta=1$ ) and a massive free theory (at $\delta=0$ ). The perturbative calculation is now done in powers of the bookkeeping parameter $\delta$ and extremized with respect to $m_{0}$ at $\delta=1$ (Principle of Minimal Sensitivity [18]). Starting with $\mathrm{O}(\delta)$ one has at $\delta=1$

$$
M_{F}^{(1)}\left(m_{0}\right)=m_{F}^{(0)}+g_{0}^{2} m_{F}^{(1)}-m_{0} \frac{\partial m_{F}^{(0)}}{\partial m_{0}}=g_{0}^{2} m_{F}^{(1)},
$$

which has no nontrivial extremum in $m_{0}$ at $T=0$ nor at finite temperatures. At $\delta=1$ the $\mathrm{O}\left(\delta^{2}\right)$ fermionic mass is given by

$$
M_{F}^{(2)}\left(m_{0}\right)=g_{0}^{2} m_{F}^{(1)}-g_{0}^{2} m_{0} \frac{\partial m_{F}^{(1)}}{\partial m_{0}}+g_{0}^{4} m_{F}^{(2)} .
$$

The extremization with respect to $m_{0}$ in the limit $\epsilon \rightarrow 0$ does not give any useful information

since the term $g_{0}^{4} m_{F}^{(2)}$, which has no nontrivial extremum, dominates. This behaviour, which persists to higher orders, has also been noted at zero temperature [5] and we find that the situation does not change at finite temperatures.

\section{THE VARIATIONAL CALCULATION TO ALL ORDERS.}

In this section we shall follow Ref. [5] to perform an all order variational calculation in the large $N$ limit eliminating the optimization problem encountered in the previous section. The general philosophy within variational methods is to start with a trial value which is expected to be reasonably close to the true value of the physical parameters. In our case this means that we can start by formulating a nonperturbative ansatz which already resums a good part of the RG behaviour of the fermionic mass before launching into the actual variational calculation. Of course this is a rather easy task within the large $N$ limit, where the exact 
answer Eq. (16) constitutes the natural choice. Performing the substitutions Eqs. (2) and (3) in Eq. (16) we get

$$
M_{F}(f)=\frac{m_{0}(1-\delta)}{f(\delta)}
$$

where we have defined

$$
f(\delta)=1-\frac{N}{2 \pi} \delta g_{0}^{2} m_{0}^{-\epsilon}(1-\delta)^{-\epsilon} f^{\epsilon}\left[(4 \pi)^{\frac{\epsilon}{2}} \Gamma(\epsilon / 2)-4 I_{1}^{T}\left(y_{F}^{\prime}\right)\right],
$$

with $y_{F}^{\prime}=M_{F}(f) / T$. It is now possible to perform an expansion in powers of $\delta$ to order- $n$ around the free theory $(\delta=0)$. Using contour integration one obtains $M_{F}$ to $n^{\text {th }}$ order of perturbation theory

$$
M_{F}^{(n)}\left(m_{0}\right)=\frac{1}{2 \pi i} \oint d z\left(\frac{1}{z}+\frac{\delta}{z^{2}}+\ldots+\frac{\delta^{n}}{z^{n+1}}\right) \frac{m_{0}(1-z)}{f(z)}
$$

which, at $\delta=1$, gives

$$
M_{F}^{(n)}\left(m_{0}\right)=\frac{1}{2 \pi i} \oint \frac{d z}{z^{n+1}} \frac{m_{0}}{f(z)}
$$

Once $Z_{m}$ and $Z_{g}$ are applied to the bare $m_{0}$ and $g_{0}^{2}$ one gets the finite expression for the dimensionless quantity $M_{F} / \Lambda_{\overline{M S}}$

$$
\frac{M_{F}^{(n)}(m)}{\Lambda_{\overline{M S}}}=\frac{1}{2 \pi i} \oint \frac{d z}{z^{n+1}} \frac{m}{\Lambda_{\overline{M S}}}\left\{1+\frac{N}{\pi} g^{2} z\left[\ln \left(\frac{M_{F}(f)}{\bar{\mu}}\right)+2 I_{1}^{T}\left(y_{F}^{\prime}\right)\right]\right\}^{-1} .
$$

As noted in the case of the anharmonic oscillator [19], it is possible to extract more structure from the limit of infinite order by rescalling $m$ with the order $n$. After distortion of the contour it is clear that only the vicinity of $z=1$ survives in the limit $n \rightarrow \infty$, which can be analyzed by changing variables

$$
1-z=\frac{v}{n}
$$

Rescalling $m$ by introducing $m^{\prime}=m / n$ we get, in the $n \rightarrow \infty$ limit

$$
\frac{M_{F}\left(m^{\prime \prime}\right)}{\Lambda_{\overline{M S}}}=\frac{1}{2 \pi i} \oint \frac{d v e^{v} m^{\prime \prime}}{K(v)}
$$


where the integration runs counterclockwise around the negative real axis. The function $K(v)$ is given by

$$
K(v)=\ln \left(\frac{m^{\prime \prime} v}{K(v)}\right)+2 I_{1}^{T}\left(\frac{m^{\prime \prime} v}{t K(v)}\right)
$$

where

$$
m^{\prime \prime}=\frac{m^{\prime}}{\Lambda_{\overline{M S}}}\left(\frac{N g^{2}}{\pi}\right)^{-1},
$$

with $t=T / \Lambda_{\overline{M S}}$. Equations (32) and (33), which summarize our variational approach, should be understood as follows: for a given variational parameter $m^{\prime \prime}$, and a given temperature $T$, Eq. (33) enables one to determine $K(v)$ self consistently. The variational result is then given in an explicitly RG invariant way by extremizing Eq. (32) with respect to $m^{\prime \prime}$. In general this program has to be achieved numerically. However, before doing that one can use the fact that the large $N$ limit is free from infra red divergences to perform an analytical exploitation of the $m^{\prime \prime} \rightarrow 0$ limit where the integral is dominated by the $v \sim 0$ region. Simple considerations show that for $T<T_{c}$

$$
K(v) \underset{m^{\prime \prime} \rightarrow 0}{\sim} m^{\prime \prime} v \frac{\Lambda_{\overline{M S}}}{M_{F}(T)}
$$

where $M_{F}(T)$ is given in Eq. (23). Moreover, $K(v)$ has a cut starting at a negative value of $v$ and lying along the negative real axis. Hence, the integral Eq. (32) converges exponentially to the expected result as $m^{\prime \prime} \rightarrow 0$. As $T \rightarrow T_{c}$, the branching point approaches the value $v=0$ merging to it at $T=T_{c}$. At this point the integral becomes divergent at $m^{\prime \prime}=0$ and does not allow more extrema for $T>T_{c}$. Numerical results obtained at different temperatures (see Fig. 1) indicate that $m^{\prime \prime}=0$ is in fact the only real extremum which leads to a smooth (second order) phase transition occuring at the critical temperature

$$
t_{c}=0.57
$$

The standard large $N$ structure of the phase transition is then exactly reproduced. 


\section{CONCLUSIONS}

In this letter, we have shown that a recently proposed variational method can be successfully generalized to the finite temperature domain. For comparison purposes, and to introduce the method in the study of phase transitions we have chosen to start with the large $N$ limit of the Gross-Neveu model. We have seen that optimization problems encountered in the zero temperature low order variational calculation persist at finite temperatures. Then, by applying the all order variational calculation scheme developed in Ref. [5] we were able to recover the usual large $N$ result for the phase transitionof the GN model, showing that the whole calculational scheme works remarkably well at all temperatures. The finite $N$ case, which is interesting due to some important theorems related to phase transitions

in one space dimension is also technically much more complex and will be discussed in a forthcoming work.

\section{ACKNOWLEDGMENTS}

M.B.P. would like to thank $\mathrm{CNPq}($ Brazil) for a post-doctoral grant. 


\section{REFERENCES}

[1] P.M. Stevenson, Phys. Rev. D30,1712(1984); A. Okopińska, ibid. D35,1835(1987); P.M. Stevenson and I. Stancu ibid. D42,2710(1990).

[2] A. Duncan and M. Moshe, Phys. Lett B215, 352(1988); H.F. Jones and M. Moshe, Phys. Lett. B234,492(1990); S.K. Gandhi, H.F. Jones and M.B. Pinto, Nucl. Phys. B359,429(1991);S.K. Gandhi and M.B. Pinto, Phys. Rev. D49,4258(1994).

[3] A. Neveu, Nucl. Phys. B18B(Supp.),242(1990)

[4] D. Gross and A. Neveu, Phys. Rev. D10,3235(1974)

[5] C. Arvanitis, F. Geniet and A. Neveu, Montpellier preprint PM 94-19 (hep-th/9506188)

[6] C. Arvanitis, F. Geniet, M. Iacomi, J.-L. Kneur and A. Neveu, Montpellier preprint PM 94-20

[7] M. B. Pinto, Phys. Rev. D50,7673(1994)

[8] L. Jacobs, Phys. Rev. D10,3956(1974)

[9] B.J. Harrington and A. Yildiz, Phys. Rev. D11,779(1974)

[10] L.D. Landau and E.M. Lifshtiz, Statistical Physics (Pergamon, N.Y., 1958) p.482; S. Coleman, Commun. Math. Phys. 31,259(1973);N.D. Mermin and H. Wagner, Phys. Rev. Lett. 17,1133(1966); R.F. Dashen, S.-K. Ma and R. Rajaraman, Phys. Rev D11,1499(1975).

[11] E. Witten, Nucl. Phys. B145,110(1978).

[12] V.L. Berezinski, Sov. Phys. Jetp 32, 493(1970); J.M. Kosterlitz and D.J. Thouless, J. Phys. C6,1181(1973).

[13] E. Abdalla, B. Berg and P. Weiz, Nucl. Phys. B157,387(1979); R. Köberle, V. Kurak and J.A. Swieca, Phys. Rev D20,897(1979), Erratum 2638. 
[14] A. D'Adda and A.C. Davis, Phys. Lett. B101,85(1981).

[15] M. Karawski and M.J. Thun, Nucl. Phys. B130,224(1977); E. Witten, Nucl. Phys. B142,285(1978); R. Shankar and E. Witten, Nucl. Phys. B141349,(1978); M. Karowski and M.J. Thun, Nucl. Phys. B190,67(1981).

[16] B. Bellet, P. Garcia, F. Geniet and M.B. Pinto; in preparation

[17] D. Bailin and A. Love, Introduction to gauge field theory (Adam Higler, Bristol, 1986); R. J. Rivers, Path Integral Methods in Quantum Field Theories(CUP, Cambridge,1987)

[18] P.M. Stevenson, Phys. Rev. D23,2916(1981)

[19] B. Bellet, P. Garcia and A. Neveu Montpellier preprints PM 94-21 (hep-th/9507155) and PM 94-22 (hep-th/9507156) 


\section{FIGURES}

FIG. 1. $M_{F}(T) / M_{F}(0)$ as a function of the arbitrary parameter $m^{\prime \prime}$ for different temperatures.

From top to bottom the curves represent $t=0.1, t=0.5$ and $t=0.55$ respectively. 